\title{
Case Report on Esophageal Coin Management at District Hospital
}

\author{
Gupta $\mathbf{S}^{\mathbf{1}^{*}}$, Sharma $\mathbf{N}^{2}$, Thakali $\mathbf{K}^{3}$ \\ ${ }^{1}$ Assistant Professor, Department of General Practice and Emergency, \\ Gandaki Medical College Teaching Hospital, Pokhara, Nepal \\ ${ }^{2}$ Health Service Director, HDCS, Nepal \\ ${ }^{3}$ Lecturer, Department of General Practice and Emergency, NAIHS, Nepal
}

\author{
Keywords \\ Coin, Esophageal foreign body, \\ District Hospital.

\section{Corresponding author} \\ Dr. Sandeep Gupta, \\ Assistant Professor \\ Department of General Practice and \\ Emergency, Gandaki Medical College \& \\ Teaching Hospital, Pokhara, Nepal \\ Email: sandeep140@hotmail.com
}

\begin{abstract}
Accidental foreign body ingestion is commonly encountered in the pediatric population, with a peak incidence between the ages of six months and three years. Since the act may go unnoticed, the child may present late. Here, we report a case of an 11 year old boy who present at Lamjung District Hospital after 24 hours of ingestions of two rupee Indian coin. To the best of our knowledge this is the first time we describe a case report on coin removal at district hospital of Nepal.
\end{abstract}

\section{INTRODUCTION}

The majority of foreign body ingestions occur in the pediatric population, accounting for $75-85 \%$ of patients with foreign bodies in the upper GI tract but edentulous adults are also at greater risk of ingesting foreign bodies, including an obstructing food bolus or their dental prosthesis ${ }^{1,2}$. Coins are the foreign body most commonly ingested in infants and children ${ }^{3}$.

Anatomically the narrowest area within the GI tract is the esophagus, making this the commonest site of foreign body impaction. Within esophagus foreign body may lodge in the thoracic inlet, the aortic arch area, or the gastro esophageal (GE) junction. Coins are the most common foreign body ingested by children; others include toys, toy parts, magnets, batteries, safety pins, screws, marbles, bones, and food boluses ${ }^{4}$.

Foreign bodies in the esophagus can produce various symptoms, including dysphagia, drooling, and occasionally airway obstruction. Gastrointestinal foreign bodies produce less specific symptoms, including abdominal pain, melena, and hematochezia. All children with a history of foreign body ingestion should be evaluated with radiographs of the neck, chest, and abdomen. Radiolucent objects require direct visualization or contrast radiographs ${ }^{4}$. We present our experience of managing an impacted coin at upper esophagus.

\section{CASE PRESENTATION}

An 11 year old boy studying in grade 5 of Lamjung District attended the emergency room of Lamjung District Community Hospital with his father with history of ingestion of coin almost after 24 hours ago. He complained of mild discomfort over his throat. The Anterior-posterior radiograph film showed an esophageal foreign body lodged at the level of the cricopharyngeus muscle (Fig 1). His vitals and systemic examination were unremarkable. We plan for admission and evaluation 
under anesthesia the next day after observing overnight at our hospital. He was given General Anesthesia and endotracheal intubation done. Under direct visualization with the help of Magill forceps the coin was grasped in between the blade of forceps. The forceps along with the coin was safely removed (Fig 2 and 3). The procedure was uneventful and with no any trauma or hemorrhage noted. After been observed over night our patient was discharged on next day with smile on his face.

Fig 1: Anterior-posterior radiograph film showed an esophageal foreign body

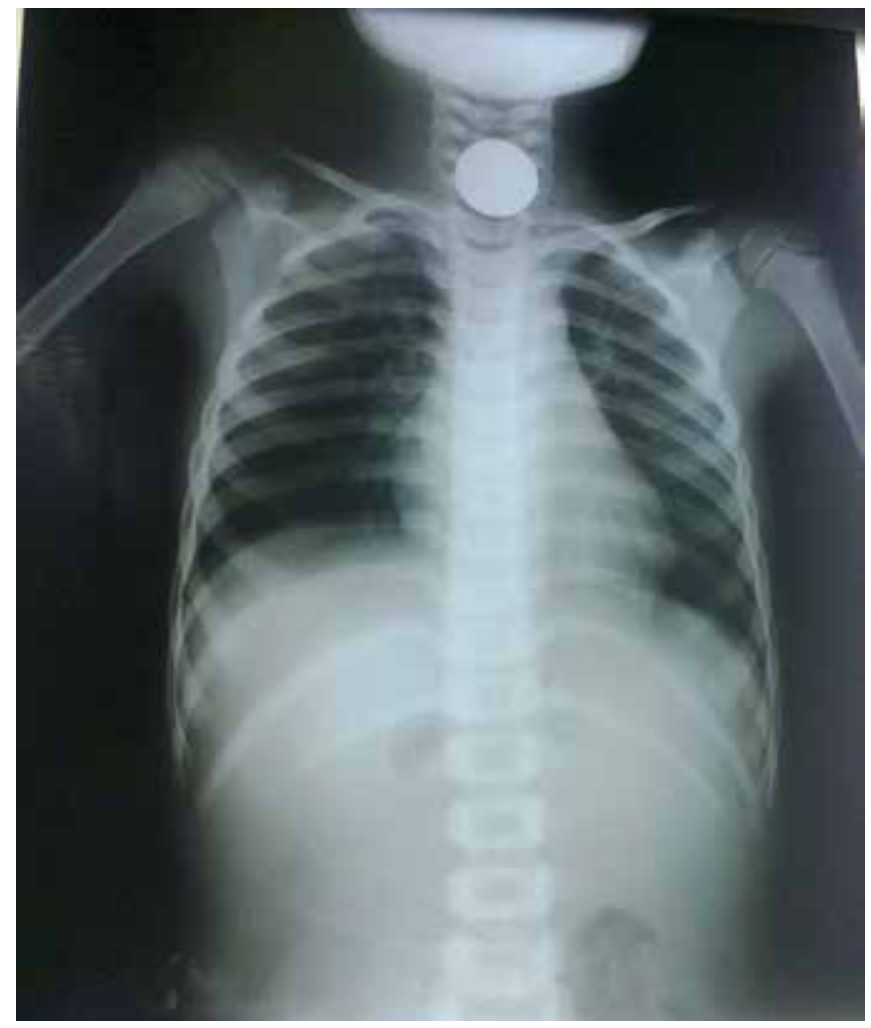

Fig 2: Foreign body been grasped with Magill forceps

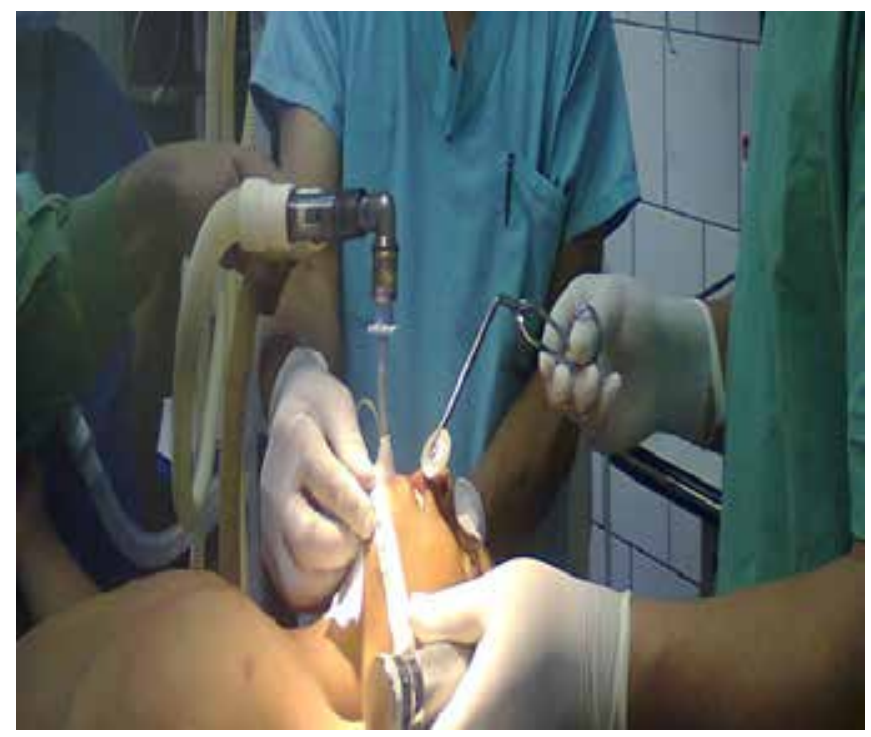

Fig 3: Foreign Body was a two rupee Indian coin

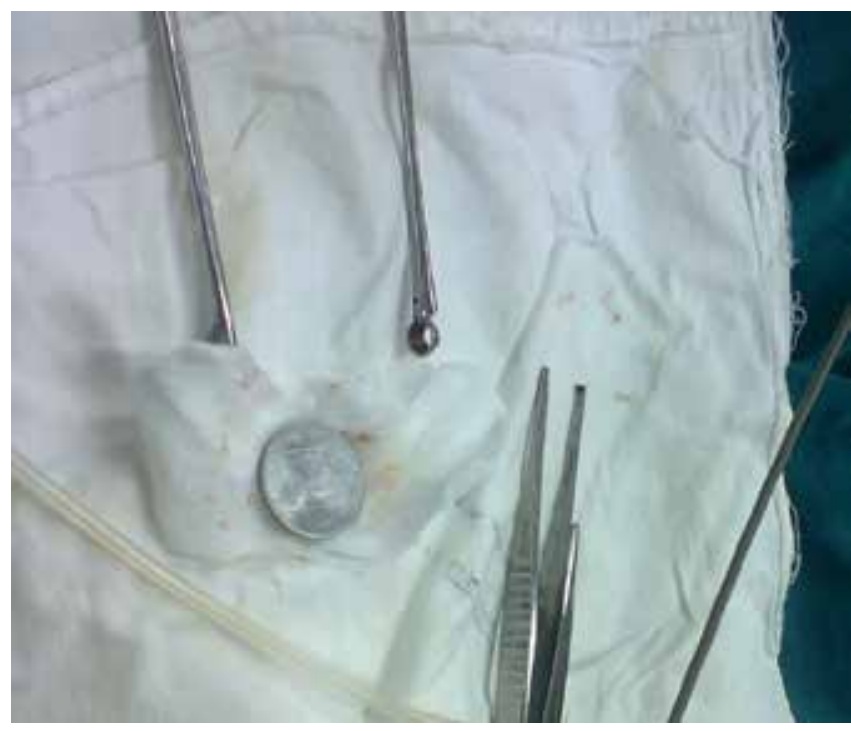

\section{DISCUSSION}

Foreign body ingestion is a potentially serious problem that peaks in children. The common sites of retained esophageal foreign bodies are related to age. Clinical management focuses on identifying and treating the cases at risk for complications, which depends on the location and type of foreign body. Children are more typically have objects entrapped in the upper part of the esophagus at the level of the cricopharyngeus muscle $70 \%$, aortic cross over $20 \%$ and lower esophageal sphincter $10 \%$ where as adults more commonly have entrapment at the lower esophageal sphincter $60 \%$, cricopharyngeus muscle $25 \%$ and aortic cross over $15 \%{ }^{5}$.

Most children with esophageal foreign bodies are brought to medical attention by their parents. A careful history and physical examination are the keystones in diagnosing an esophageal foreign body and to the prevention of its complications. Imaging can be used to confirm the findings and to localize the site of the foreign body. The diagnostic steps and treatment depend on the patient's symptoms, the shape and location of the foreign body, whether it is radio-opaque, or whether it has magnetic properties ${ }^{5}$.

Airway and breathing should always be examined first. The physical examination of the neck may reveal swelling, erythema, or crepitus, suggesting that an esophageal perforation has occurred, and surgical consultation is mandatory. The chest examination may reveal inspiratory stridor or expiratory wheezing, suggesting a lodged esophageal foreign body with tracheal compression. The abdominal examination may show evidence of small bowel obstruction or perforation, in which case 
immediate surgical consultation and abdominal imaging should be obtained ${ }^{6}$.

A variety of techniques are used to extract foreign bodies from the esophagus or stomach. Rigid esophagoscopy and flexible endoscopy for most foreign body extractions have been used at specialized centers. Magill forceps, Bougienage (passage of a dilator) and Foley catheter has been used in some of the centers to removed foreign bodies like coins ${ }^{7}$. In our study as well we used the Magill forceps to grasp and extract the coin from the impacted in the upper esophagus.

\section{REFERENCES}

1. Uyemura MC. Foreign body ingestion in children. Am Fam Physician. 2005; 72: 287-91.

2. Singh G, SharmaS, KhuradeS, GooptuS. Ingested foreign bodies in children: A report of two cases. Journal of Family Medicine and Primary Care. 2014; 3(4): 452455. doi:10.4103/2249-4863.148148.
3. Heinzerling NP, Christensen MA, Swedler R, et al. Safe and effective management of esophageal coins in children with bougienage. Surgery. 2015 Jul 31. pii: S0039-6060(15)00524-3. doi: 10.1016/j.surg.

4. Arana A, Hauser B, Hachimi-Idrissi S, Vandenplas Y. Management of ingested foreign bodies in childhood and review of the literature. Eur J Pediatr. 2001; 160:468.

5. Krishnamurthy A, Ramshankar V. The vulnerable Indian one rupee coin. J Family Med Prim Care. 2013; 2: 381-3.

6. Katsinelos P, Kountouras J, Paroutoglou G, et al. Endoscopic techniques and management of foreign body ingestion and food bolus impaction in the upper gastrointestinal tract: A retrospective analysis of 139 cases. J Clin Gastroenterol. 2006; 40: 784.

7. Arana A, Hauser B, Hachimi-Idrissi S, Vandenplas Y. Management of ingested foreign bodies in childhood and review of the literature. Eur J Pediatr. 2001; 160: 468. 